\title{
Hybrid Segmentation of Anatomical Data
}

\author{
Celina Imielinska ${ }^{1}$, Dimitris Metaxas, ${ }^{2}$ Jayaram Udupa ${ }^{3}$, Yinpeng $\mathrm{Jin}^{4}$, Ting $\mathrm{Chen}^{2}$, \\ ${ }^{1}$ College of Physicians and Surgeons, Office of Scholarly Resources, \\ Medical Informatics Dept. and Dept. of Computer Science, Columbia University \\ ci42@columbia.edu \\ ${ }^{2}$ Dept. of Computer and Information Science, University of Pennsylvania \\ ${ }^{3}$ Medical Image Processing Group, Dept. of Radiology, University of Pennsylvania \\ ${ }^{4}$ Department of Biomedical Engineering, Columbia University
}

\begin{abstract}
We propose new hybrid methods for automated segmentation of radiological patient data and the Visible Human data. In this paper, we integrate boundary-based and region-based segmentation methods which amplifies the strength but reduces the weakness of both approaches. The novelty comes from combining a boundary-based method, the deformable model-based segmentation with region-based segmentation methods, the fuzzy connectedness and Voronoi Diagram-based segmentation, to develop hybrid methods that yield high precision, accuracy and efficiency. This work is a part of a NLM funded effort to provide a fully implemented and tested Visible Human Project Segmentation and Registration Toolkit (Insight).
\end{abstract}

\section{Introduction}

In this paper we develop and test new hybrid methods for segmenting radiological (patient) data (e.g. CT, MRI, PET) and the Visible Human data. The novelty stems from the integration of deformable model-based segmentation methods with a variety of region-based methods which aims toward the development of segmentation methods that yield high precision, accuracy and efficiency. This is a collaborative project between the University of Pennsylvania and Columbia University, a part of a larger effort, funded by NLM, to provide the Visible Human Project Segmentation and Registration Toolkit (Insight, http://visual.nlm.nih.gov/insight/).

We have developed powerful region and contour-based segmentation tools that we plan to further extend to meet the above goals [5],[7], [8], [9], [17], [18]. Radiological image data of internal organs are routinely used at the University of Pennsylvania, where the Interactive Virtual Environment for Modeling Anatomy and Physiology has been developed, in addition to 3DVIEWNIX, [18], a Unix-based software system for the visualization, manipulation, and analysis of multidimensional multi-parametric, multi-modality images. Highly detailed segmentations and 3D visualizations of the Visible Human dataset are used in interactive applications, such as the Vesalius ${ }^{\mathrm{TM}}$ Project at Columbia University, an interdisciplinary effort to create an environment for anatomy and other medical applications [6]. Integration of boundary-based and region-based segmentation methods amplifies the strengths but reduces the weaknesses of both approaches. 


\section{Approach and Methodology}

Automatic internal organ segmentation from various imaging modalities is a very important yet open research problem. Over the past several years, a variety of segmentation methods have been developed. Boundary-based techniques such as snakes [11] start with a deformable boundary and attempt to align this boundary with the edges in the image. The solution to these systems generally involves minimizing an energy functional which quantifies the shape of the model and image information near the boundary of the model. To avoid becoming stuck in local minima, most model-based techniques require that the model be initialized near the solution. User steered methods such as live wire that are used in day-to-day clinical research find a global optimum through dynamic programming. Region-based or statistical techniques such as region growing [3],[7] or MAP-based methods [2] assign membership to objects based on homogeneity statistics. The advantage here is that image information inside the object is considered as well as on the boundaries. However, in the region based framework, there is no provision for including the shape of the region in the decision making process, which can lead to noisy boundaries and holes in the interior of the object.

Like several other recent approaches [4],[20], our design integrates the boundary and region-based techniques into a hybrid framework. By combining these techniques, hybrid approaches offer greater robustness than either technique alone. However, most previous work still requires significant initialization to avoid local minima. Furthermore, most of the earlier approaches use prior models for their region-based statistics, which we would rather avoid to increase usefulness in situations where a comprehensive set of priors may not be available.

We have recently developed a new approach to internal organ segmentation that is based on the integration of region-based and physics-based boundary estimation methods [8],[9]. Starting from a single pixel within the interior of an object, we make an initial estimate of the object's boundary using the fuzzy connectedness method [17] and clustering. A deformable surface model is then fitted to the extracted boundary data to fill in the missing boundary data and to override the spurious boundary data due to image noise. This is achieved by generalizing the formulation of our deformable models [12] to incorporate simple domain-specific knowledge.

In this paper we will further develop this approach by integrating deformable models with fuzzy connectedness, and the region-based color segmentation method developed at Columbia [7]. In addition, we will present a method based on an integration of Markov Random Field (Gibbs prior) and deformable models. In particular we develop two types of automatic segmentation algorithms: 1) Hybrid Method I: Integration of fuzzy connectedness, Voronoi Diagram; 2) Hybrid Method II: Integration of Gibbs prior and deformable models.

\section{Hybrid Method I: Integration of Fuzzy Connectedness and Voronoi Diagram}

We present a hybrid segmentation method which requires minimal manual initialization, where we integrate the fuzzy connectedness, and Voronoi Diagram 
(VD). We will start with fuzzy connectedness algorithm to generate a region with a sample of tissue which we plan to segment. From the sample region, we will generate automatically homogeneity statistics for the VD-based algorithm which will produce an estimation of the boundary in a number of iterations. We will give an overview of the component algorithms used in the hybrid segmentation algorithm below.

\subsection{Fuzzy Connectedness Algorithm.}

This method described in detail in [17], uses the fact that medical images are inherently fuzzy. We define affinity between two elements in an image (e.g. pixels, voxels, spels ) via a degree of adjacency and the similarity of their intensity values. The closer the elements are and more similar their intensities are, the greater is the affinity between them. There are two important characteristics of a medical image. First, it has graded composition coming from material, blurring, noise and background variation. Second, the image elements that constitute an anatomical object hang together in a certain way. Both these properties, graded composition and hanging togetherness are fuzzy properties. The aim of fuzzy connectedness is to capture the global hanging togetherness using image-based local fuzzy affinity.

Let us define a scene over a digital space $\left(Z^{n}, \alpha\right)$ as a pair $\Omega=(C, f)$, where $\mathrm{C}$ is an ndimensional array of spels (elements) and $f: C \rightarrow[0,1]$. Fuzzy affinity $\kappa$ is any reflexive and symmetric fuzzy relation in $\mathrm{C}$, that is:

$$
\begin{aligned}
& \kappa=\left\{\left((\mathrm{c}, \mathrm{d}), \mu_{\kappa}(\mathrm{c}, \mathrm{d})\right) \mid \mathrm{c}, \mathrm{d} \in \mathrm{C}\right\} \\
& \mu_{\kappa}: \mathrm{C} \times \mathrm{C} \rightarrow[0,1] \\
& \mu_{\kappa}(\mathrm{c}, \mathrm{c})=1, \text { for all } \mathrm{c} \in \mathrm{C} \\
& \mu_{\kappa}(\mathrm{c}, \mathrm{d})=\mu_{\kappa}(\mathrm{d}, \mathrm{c}), \text { for all } \mathrm{c}, \mathrm{d} \in \mathrm{C} .
\end{aligned}
$$

The general form of $\mu_{\kappa}$ can be written as follows. For all $\mathrm{c}, \mathrm{d} \in \mathrm{C}$,

$$
\mu_{\kappa}(\mathrm{c}, \mathrm{d})=\mathrm{g}\left(\mu_{\alpha}(\mathrm{c}, \mathrm{d}), \mu_{\psi}(\mathrm{c}, \mathrm{d}), \mu_{\phi}(\mathrm{c}, \mathrm{d}), \mathrm{c}, \mathrm{d}\right)
$$

where: $\mu_{\alpha}(\mathrm{c}, \mathrm{d})$ represents the degree of adjacency of $\mathrm{c}$ and $\mathrm{d} ; \mu_{\psi}(\mathrm{c}, \mathrm{d})$ represents the degree of intensity homogeneity of $\mathrm{c}$ and $\mathrm{d} ; \mu_{\phi}(\mathrm{c}, \mathrm{d})$ represents the degree of similarity of the intensity features of $\mathrm{c}$ and $\mathrm{d}$ to expected object features. Fuzzy $\kappa-$ connectedness $\mathrm{K}$ is a fuzzy relation in $\mathrm{C}$, where $\mu_{K}(\mathrm{c}, \mathrm{d})$ is the strength of the strongest path between $\mathrm{c}$ and $\mathrm{d}$, and the strength of a path is the smallest affinity along the path. To define the notion of a fuzzy connected component, we need the following hard binary relation $\mathrm{K}_{\theta}$ based on the fuzzy relation $\mathrm{K}$. Let $\Omega=(C, f)$ be a membership scene over a fuzzy digital space $\left(Z^{n}, \alpha\right)$, and let $\kappa$ be a fuzzy spel affinity in $\Omega$. We define a (hard) binary relation $\mathrm{K}_{\theta}$ in $\mathrm{C}$ as

$$
\mu_{\mathrm{K}_{\theta}}(c, d)=\left\{\begin{array}{cc}
1, & \text { iff } \mu_{\kappa}(c, d) \geq \theta \in[0,1] \\
0, & \text { otherwise }
\end{array}\right.
$$

Let $\mathrm{O}_{\theta}$ be an equivalence class [14, Chap.10] of the relation $\mathrm{K}_{\theta}$ in C. A fuzzy $\kappa$ component $\Gamma_{\theta}$ of $C$ of strength $\theta$ is a fuzzy subset of $\mathrm{C}$ defined by the membership function

$$
\mu_{\Gamma_{\theta}}= \begin{cases}f(c), & \text { iff } c \in \mathrm{O}_{\theta} \\ 0, & \text { otherwise }\end{cases}
$$


The equivalence class $\mathrm{O}_{\theta} \subset \mathrm{C}$, such that for any $\mathrm{c}, \mathrm{d} \in \mathrm{C}, \mu_{\kappa}(\mathrm{c}, \mathrm{d}) \geq \theta, \theta \in[0,1]$, and for any e $\in \mathrm{C}-\mathrm{O}_{\theta}, \mu_{\kappa}(\mathrm{c}, \mathrm{d})<\theta$. We use the notation $[o]_{\Theta}$ to denote the equivalence class of $\mathrm{K}_{\theta}$ that contains $O$ for any $O \in \mathrm{C}$. The fuzzy $\kappa$-component of $C$ that contains $O$, denoted $\Gamma_{\theta}(O)$, is a fuzzy subset of $\mathrm{C}$ whose membership function is

$$
\mu_{\Gamma_{\theta}(o)}= \begin{cases}f(c), & \text { iff } c \in[o]_{\theta} \\ 0, & \text { otherwise }\end{cases}
$$

A fuzzy $\kappa \theta$-object of $\Omega$ is a fuzzy $\kappa$-component of $\Omega$ of strength $\theta$. For any spel $O \in$ C, a fuzzy $\kappa \theta$-object of $\Omega$ that contains $O$ is a fuzzy $\kappa$-component of $\Omega$ of strength $\theta$ that contains $O$.Given $\kappa, O, \theta$, and $\Omega$, a fuzzy $\kappa \theta$-object of $\Omega$ of strength $\theta \in[0,1]$ containing $O$, for any $O \in \mathrm{C}$, can be computed via dynamic programming [17].

\subsection{Voronoi Diagram(VD)-Based Algorithm}

This algorithm, which is described in detail in [7], is based on repeatedly dividing an image into regions using VD and clasifying the Voronoi regions based on a selected homogeneity classifier for the segmented anatomical tissue. We will use the algorithm as a component in the hybrid method where the classifiers for different tissue type will be generated automatically from the region segmented by the fuzzy connectedness method. VD and Delaunay triangulation (DT) play a central role in the algorithm. In 2D, the VD for a set $\mathrm{V}$ of points is a partition of the Euclidean plane into Voronoi regions of points closer to one point of $\mathrm{V}$ than to any other seed point [14]. For any $p_{i} \in V, V=\left\{p_{1}, \ldots, p_{n}\right\}$,

$$
V D\left(p_{i}\right)=\left\{x \in R^{2} \mid d\left(x, p_{i}\right) \leq d\left(x, p_{j}\right), \forall j \neq i, 1 \leq j \leq n\right\}
$$

Similarly, we define the VD in 3D:

$$
V D\left(p_{i}\right)=\left\{x \in R^{3} \mid d\left(x, p_{i}\right) \leq d\left(x, p_{j}\right), \forall j \neq i, 1 \leq j \leq n\right\}
$$

Two Voronoi regions are adjacent if they share a Voronoi edge. The DT, of V is a dual graph of the Voronoi diagram of $\mathrm{V}$, obtained by joining two points whose Voronoi regions are adjacent.

\subsection{The Hybrid Method I}

The algorithm integrates two methods, the fuzzy connectedness and VD-based algorithm. We will outline the algorithm first, and explain the component steps later. The fuzzy connectedness algorithm is used to segment a fragment of the target tissue. From the sample, a set of statistics is generated automatically, in RGB and HVC color spaces, to define the homogeneity operator. The homogeneity operator will be used as a multi-channel classifier for the VD-based algorithm. As we mentioned, we will use, in the future, the deformable model, to determine the final (3D) smooth boundary of the segmented region. Below, we outline the hybrid method: 
Step 1. We run the fuzzy connectedness algorithm to segment a sample of the target tissue, and generate statistics, average and variance, in three "strong" color channels, in two color spaces, RGB and HVC.

Step 2. Run the VD-based algorithm using multiple color channels, until it converges: (a) For each Voronoi region, classify it as interior/exterior/boundary region using multi-channel homogeneity operator; (b) Compute DT and display segments which connect boundary regions; (c) Add seeds to Voronoi edges of Voronoi boundary regions; (d) GoTo Step 2(a) until the algorithm converges to a stable state or until the user chooses to quit.

Step 3. (future) Use the deformable model to determine the final (3D) boundary and re-set the homogeneity operator.

Implementation of Step 1. To initialize the fuzzy connectedness algorithm and establish the mean and standard deviation of spel values and their gradient magnitudes. The user collects the pixels within the region of interest, by clicking on the image and selecting at each time a square region with $7 \times 7$ pixels. Then an initial seed spel is selected to compute the fuzzy connectedness, using the dynamic programming approach [17]. We determine the strength of the fuzzy connectedness $\theta$, $\theta \in[0,1]$, by letting the user to select interactively its threshold value, such that the initially segmented sample of the target tissue resembles the shape of the underlying image. For a binary image with a roughly segmented sample of a tissue, we generate the "strongest" three channels in two color spaces, RGB and HVC [15], for average and variance, respectively. First, we define for the binary image, the smallest enclosing rectangle, a region of interest (ROI), in which we identify the segmented image and its background. Within the ROI, we calculate the mean and variance in each of the six color channels $(\mathrm{R}, \mathrm{G}, \mathrm{B}, \mathrm{H}, \mathrm{V}, \mathrm{C})$ for the object and its background, respectively. Then three channels with the largest relative difference in mean value and in variance value between the object and its background are selected, respectively. The homogeneity operator for the VD-based algorithm uses the expected mean/variance values of the object together with tolerance values, computed for each selected channel, for classifying the internal and external region

Implementation of Step 2. We build an initial VD by generating some number of random seed points (Voronoi points) and then run the QuickHull, [1], to calculate the VD. Once the initial VD has been generated, the program visits each region to accumulate classification statistics and makes a determination as to the identity of the region. For each Voronoi region, the mean/variance value for the preselected channels are computed, if they are similar, then it is marked as internal, otherwise external. Those external regions that have at least one internal neighbor are marked as boundary. Each boundary region is divided for next iterations until the total number of pixels within it is less than a chose number.

\section{Hybrid Method II: Integration of Gibbs Prior and Deformable Models}

Most medical images are Markov Random Field (MRF) images, that is, the statistics of a pixel is related to the statistics of pixels in its neighborhood. According to the Equivalent Theorem proved by Hammersley and Clifford, the MRF is equivalent to 
the Gibbs field under certain conditions. Thus for medical images which are MRF images, their joint distribution can be written in the Gibbsian form, which is shown as follows.

$$
\Pi(x)=Z^{-1} \exp (-H(x)), Z=\sum_{z \in X} \exp (-H(z))
$$

where $H(x)$ represents the energy function of image $x, X$ is the set of all possible configuration of the image, $Z$ is the normalization factor or partition function in the terminology of statistical mechanics. The local and global properties of images will be incorporated into the model by designing an appropriate energy function. The lower the value of the energy function, the higher the value of the Gibbs distribution, the better the image is fit to the prior distribution. We began the establishment of the Gibbs model by designing the energy function as

$H(X)=H_{1}(X)+H_{2}(X)+H_{3}(X)$

$H_{1}(X)$ models the piecewise homogeneity statistics and $H_{2}(X)$ models the continuity of the boundary, $H_{3}(X)$ is the noise model term. For $H_{2}(X)$ we use the 3 by 3 clique. Local characteristics in the clique are given different weight. The local characteristics that more likely to appear in the image has lower weight. So when the energy function is minimized, such kind of local characteristics will increase. This will help to locate the boundary if the difference between object and background is not so big. A set of suitable parameters will enable the Gibbs prior model to: (a) Find the boundary according to the variance between objects and background. ( $\left.H_{1}(X)\right)$; (b) If the variance is not large enough or the object gradually turns into the background, $H_{2}(X)$ is capable of finding a smooth and continuous boundary estimation; (c) Erase the noise using term $H_{3}(X)$.

We use the iterated conditional mode (ICM) method to minimize the energy function. This will give an estimation of the object boundary which can be used by the deformable model. Our deformable model is a superellipsoid with local deformations. The global parameter of the model is its natural shape, and the local deformations determine its displacement from the natural shape for discrete nodes on the surface of the model. Given the reference shape $\mathbf{s}$ and displacement $\mathbf{d}$, points on a model $\mathbf{p}$ are defined by:

$$
\mathbf{p}=\mathbf{s}+\mathbf{d}
$$

To keep the continuity of the model surface, we impose a $C^{0}$ continuous deformation strain energy on it.

$\varepsilon_{m}(d)=\int \omega_{10}\left(\frac{\partial d}{\partial u}\right)^{2}+\omega_{01}\left(\frac{\partial d}{\partial v}\right)^{2}+\omega_{00} d^{2} d u$,

where $d$ is the node local deformation, $\omega_{00}$ controls the local magnitude and $\omega_{10}$, $\omega_{01}$ control the local variation of the deformation. We can calculate the stiffness matrix $\mathbf{K}$ of the model based on the strain energy. The model nodes will move under the influence of internal and external forces. The internal forces constrain the deformation of a model according to the stiffness matrix to maintain its natural shape, 
while the external forces make the model expand until they meet a balance with the internal forces. The dynamics can be described by the first order Lagrangian method:

$$
\mathrm{d}+\mathrm{Kd}=\mathrm{f} \text {, }
$$

where $f$ is the summation of external forces and boundary forces. The nodes of the model are forced outward in the direction of their normal vector. So the model will expand like a balloon being filled with gas. When the model is reaching the estimated boundary, it will also move under the influence of boundary forces, which is in the opposite direction of the balloon force. Thus the model will fit to the estimated boundaries. When the shape of the object is complicated such as at sharp corners, new nodes will be added to the model. The Finite Element Analysis (FEA) method is used in our work to calculate the deformation on each node. The deformation is updated using Euler step:

$$
d_{n+1}=d_{n}+t^{*} d
$$

where $t$ is the time step. When most nodes (>90\%) on the surface stop moving, the model stops. When the model is fitted to the estimated boundaries, we do statistics in the model region, updating the parameters for the Gibbs Prior model according to the result. The mean intensity, standard error of the object and the threshold of boundary existance will be recalculated. Weights of the local characteristics will be changed according to the global shape of the model.

The whole method can be summarized as follows: (Step 1) First create the Gibbs prior models using parameters calculated according to statistics result over a small region inside the object; (Step 2) Use the Gibbs prior models to estimate the boundaries of objects; (Step 3) Fit the deformable models to this data according to the combination of balloon and the boundary forces. (Step 4) Recalculate the parameters for the Gibbs prior model according to the results of Step3. Then go back to Step 2.

\section{Hybrid Method I: Results}

In this section we present the results from experiments using Hybrid Method I. We tested different tissue types: muscle tissue (VH data, Fig.1.) and brain tissue (VH, Fig.2, and MRI patient data, Fig.3. In Fig.1, we segment temporalis muscle, a structure in the head region. In Fig.2, we segment brain gray matter. We would like to note that we have used fuzzy connectedness extensively, in 3D, to segment gray and white matter in patient image data [19]. Gray matter and white matter are fuzzy connected entities in $3 \mathrm{D}$. With a few seed points they can be, and they are, routinely segmented.

We show that the Hybrid I algorithm can generate automated statistical homogeneity classifiers for a variety of human tissue. We tested the method with the Visible Human data and as well with a sample of MRI patient data. The method will be formally validated, in the future using our rigorous validation methodology. 


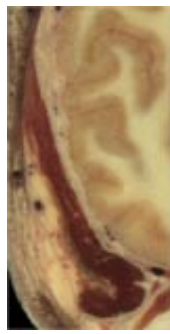

(a)

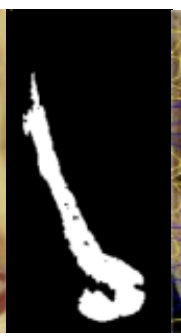

(b)

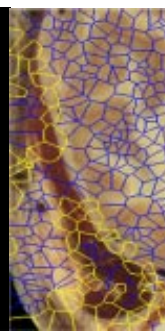

(c)

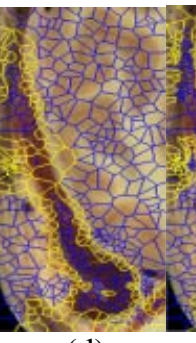

(d)

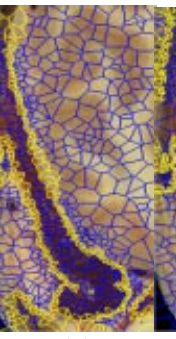

(e)

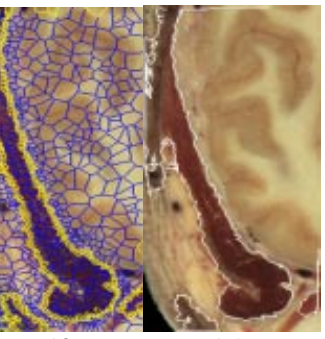

(f)

(g)

Fig.1. Hybrid Method I (segmentation of temporalis muscles): (a) Color VH male cryosection slice, (b) a fuzzy connected component, (c)-(f) iterations of the VD-based algorithm, (g) an outline of the boundary.

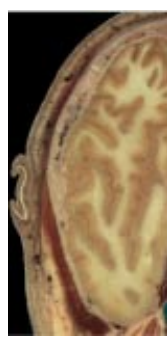

(a)

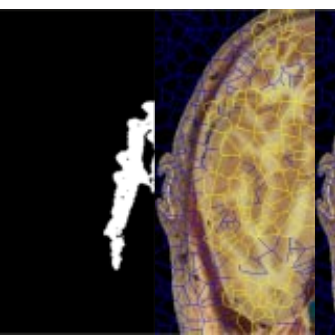

(b)

(c)

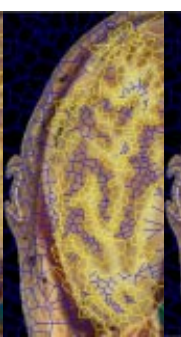

(d)

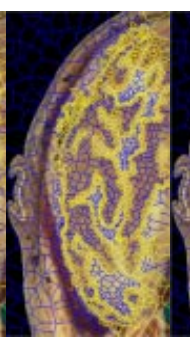

(e)

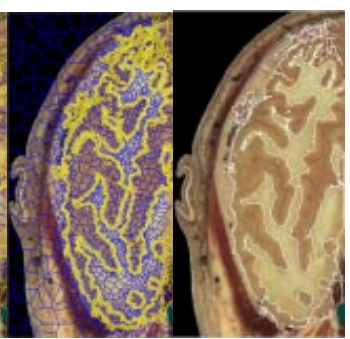

(f)

(g)

Fig.2. Hybrid Method I (segmentation of brain gray matter): (a) Color VH male cryosection slice, (b) a fuzzy connected component, (c)-(f) VD-based algorithm, (g) an outline of the boundary.

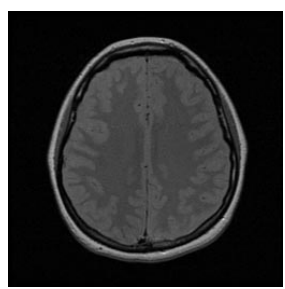

(a)

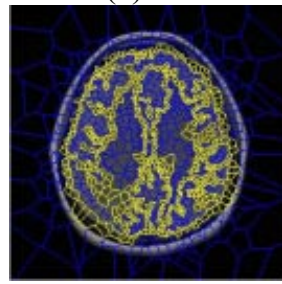

(e)

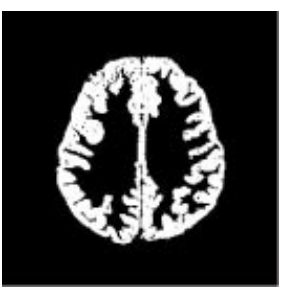

(b)

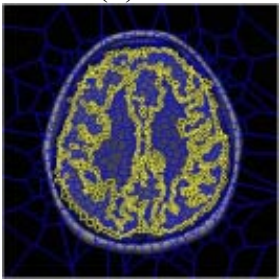

(f)

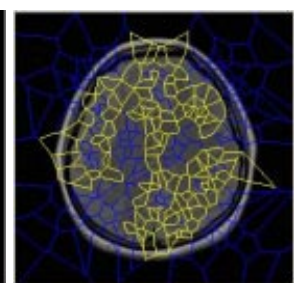

(c)

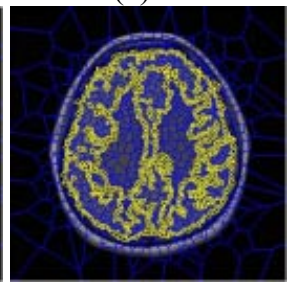

(g)

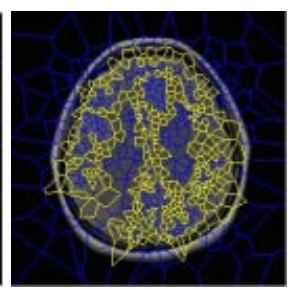

(d)

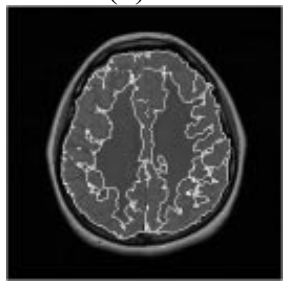

(h)

Fig.3. Hybrid Method I (segmentation of (MRI) gray matter): (a) MRI patient slice, (b) fuzzy connected component, (c)-(g) iterations of the VD-based algorithm, (h) an outline the boundary. 


\section{Hybrid Method II: Results}

We segmented below, using Hybrid Method II, structures using the VH data (rectus muscle, Fig.4., and eyeball, Fig.5) and MRI patient data (white brain matter, Fig.6). In this method, by using the Gibbs prior, we generate a better estimation of the boundary and it is used as an initialization for the deformable model. We show that Gibbs prior method can estimate the boundary well in a variety of. The deformable model provides updated parameters to the Gibbs prior model, and the iterative algorithm is applied recursively until a refined segmentation is obtained. The Hybrid Method II, will be validated, in the future, using our rigorous validation methodology.

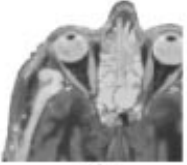

a)

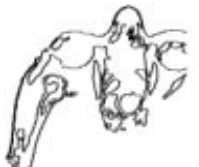

b)

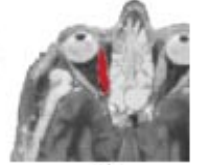

c)

Fig.4. Hybrid Method II (segmentation of rectus muscle): (a) VH slice, (b) Gibbs prior estimation (c) deformable model result.

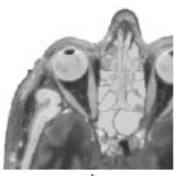

a)

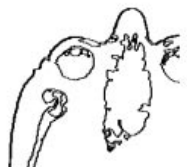

b)

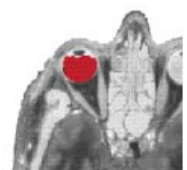

c)

Fig.5. Hybrid Method II (segmentation of eyeball (small scale)) : (a) VH slice, (b) Gibbs prior estimation (c) deformable model result.

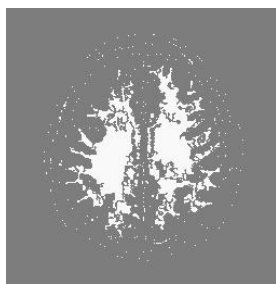

(a)

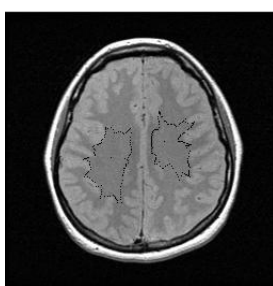

(b)

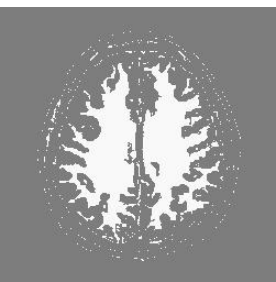

(c)

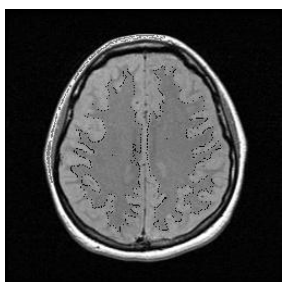

(d)

Fig.6. Hybrid Method II (segmentation of white matter from Fig. 3(a): (a)(c) Gibbs prior estimation, (b)(d) deformable model result.

Acknowledgements. This work was supported in part by NLM contract on the "VHP Segmentation and Registration Toolkit" - NLM99-103/DJH.

\section{References}

1. Barber, C.B.; Dobkin, D.P, Huhdanpaa H.:The Quickhull Algorithm for Convex Hull, The Geometry Center, University of Minnesota, 1995.

2. Blake, A., Zisserman, A.: Visual Reconstruction. MIT Press, 1987. 
3. Bertin, E., Parazza, F.; Chassery, J.M., Segmentation and Measurement Based on 3D Voronoi Diagram: Application to Confocal Microscopy, J. CMIG, 1993, 17(3), p.175-182.

4. Chakraborty, A., Duncan, J.S.: Integration of Boundary Finding and Region-Based Segmentation Using Game Theory, In Y. Bizais et al., editor, Information Processing in Medical Imaging, Kluwer, 1995, p.189-201.

5. Falcao, A., Udupa, J.K., Samarasekera, S., Sharma, S., Hirsch, B.E.; Lotufo, R.: "UserSteered Image Segmentation Paradigm: Live wire and Live Lane", Graphical Models and Image Processing, vol.60, pp.233-260, 1998.

6. http://cpmcnet.columbia.edu/vesalius

7. Imielinska, C., Downes, M., Yuan, W.: Semi-Automated Color Segmentation of Anatomical Tissue, J. of CMIG ., 24(2000), 173-180, April, 2000.

8. Jones, T, Metaxas, D.: Automated 3D Segmentation Using Deformable Models and Fuzzy Affinity, Proc. XVth Intern. Conf. on Image Processing in Medical Imaging, June 1997.

9. Jones, T., Metaxas D,: Image Segmentation based on the Integration of Pixel Affinity and Deformable Models, Proc. IEEE CVPe, Santa Barbara, CA, June 1998.

10. Kaufmann, A.: Introduction to the Theory of Fuzzy Subsets, Vol. I, Academic Press, New York, 1975.

11. Kass, M., Witkin, A., Terzopoulos, D.: Snakes: Active Contour Models, Intl. J. of Computer Vision, 1998, 1(4), p.321-331.

12. Metaxas, D., Physics-Based Deformable Models: Applications to Computer Vision, Graphics and Medical Imaging, Kluwer-Academic Publishers, 1996.

13. Panel on "Technical Challenges" at the Second User Conference of the National Library of Medicine's Visible Human Project, Oct. 1-2nd, 1998, Bethesda, MD.

14. Preparata, F.P.; Shamos, M.I.: Computational Geometry, New York, Springer, 1985.

15. Gong,Y., Sakauchi, M.: Detection of Regions Matching Specified Chromatic Features, Computer Vision and Image Understanding, 61(2), p.263-269, 1995.

16. Udupa, J., Odhner, D., Samarasekera, S., Goncalves, R., Iyer, K., Venugopal, K., Furuie, S.: 3D VIEWNIX: An Open, Transportable, Multidimensional, Multimodality, Multiparametric Imaging Software System”, SPIE Proceedings, 2164:58-73, 1994.

17. Udupa, J.K., Samarasekera, S.: Fuzzy Connectedness and Object Definition, In SPIE Proceedings, vol.2431, pp. 2-11, 1995.

18. Udupa, J.K., Samarasekera, S.: Fuzzy Connectedness and Object Definition: Theory, Algorithms, and Applications in Image Segmentation, Graphical Models and Image Processing, 58(3), pp.246-261, 1996.

19. Udupa, J.K., Wei L., Samarasekera, S.: Miki, Y.; van Buchem, M.A.; Grossman, R.I.; Multiple sclerosis lesion quantification using fuzzy Connectedness principles, IEEE Trans, Med Imaging, vol. 16, pp. 598-609,1997.

20. Zhu, S.C, Lee, T.S., Yuille, A.L.: Region Competition: Unifying Snakes, Region Growing, and Bayes/mdl for Multi-Band Image Segmentation, In Proc. Intl. Conf. on Computer Vision, 1995, p.416-423. 